\title{
A REMARK ON STRONG MAXIMUM PRINCIPLE FOR PARABOLIC AND ELLIPTIC SYSTEMS
}

\author{
XUEFENG WANG
}

(Communicated by Barbara L. Keyfitz)

\begin{abstract}
We give a strong maximum principle for some nonlinear parabolic and elliptic systems with convex invariant regions. We also obtain a version of the Hopf boundary lemma for the systems.
\end{abstract}

\section{INTRODUCTION}

The parabolic systems considered in this paper are of the form (*)

$$
\frac{\partial u}{\partial t}-D(x, t, u) \sum_{i, j=1}^{n} a_{i j}(x, t, u) \frac{\partial^{2} u}{\partial x_{i} \partial x_{j}}+\sum_{i=1}^{n} M_{i}(x, t, u) \frac{\partial u}{\partial x_{i}}=f(x, t, u)
$$

on $\Omega \times(0, T)$, where

$$
u=\left(\begin{array}{c}
u_{1} \\
\vdots \\
u_{m}
\end{array}\right),
$$

$\Omega$ is a domain in $\mathbf{R}^{n}, D(x, t, u)$, and $M_{i}(x, t, u)(i=1,2, \ldots, n)$ are $m \times m$ matrix-valued functions on $\Omega \times(0, T) \times R^{m}, a_{i j}(x, t, u) \quad(i, j=$ $1, \ldots, n)$ are real-valued functions.

Under the hypothesis that the differential operator on the left-hand side of (*) is locally uniformly parabolic on $\Omega \times(0, T)$, that $(*)$ has a $C^{2}$ convex invariant region $S \subset \mathbf{R}^{m}$, and under some regularity conditions, we show that, for $(*)$, Weinberger's version of strong maximum principle holds, which says that if there exists $a\left(x^{*}, t^{*}\right) \in \Omega \times(0, T)$ such that $u\left(x^{*}, t^{*}\right) \in \partial S$, then $u\left(\Omega \times\left(0, t^{*}\right]\right) \subset \partial S$. Moreover, if in addition that $\Omega$ satisfies the interior sphere condition, we prove that a version of the Hopf boundary lemma holds for $(*)$.

The weak and strong maximum principle for the case that in $(*), D(x, t, u)$ $\equiv I$ and $M_{i}(i=1, \ldots, n)$ are real-valued functions have been studied by Weinberger [1], the boundary point lemma, however, was not mentioned in [1]

Received by the editors February 14, 1989.

1980 Mathematics Subject Classification (1985 Revision). Primary 35B50. 
(see the main theorem in $\S 3$ ). Our basic method is the same as Weinberger's. The local defining functions of $\partial S$ plays an important role in [1] for strong maximum principle. Instead of choosing a general defining function as in [1], we prefer the distance function of $\partial S$, making the proofs more geometric.

An extension of the boundary lemma was found by W. Troy [4] for nonnegative solution of the elliptic system

$$
\sum_{i, k=1}^{n} a_{j k}^{i}(x) \frac{\partial^{2} u_{i}}{\partial x_{j} \partial x_{k}}+\sum_{j=1} b_{j}^{i}(x) \frac{\partial u_{i}}{\partial x_{j}}+\sum_{j=1}^{n} C_{i j}(x) u_{j}=0
$$

on $\Omega$, where $i=1, \ldots, m . C_{i j}(x) \geq 0$ on $\Omega$ for $i \neq j, 1 \leq i, j \leq m$.

The weak maximum principle for $(*)$ has also been studied by K. N. Chueh, C. C. Conley, and J. Smoller [2]. Their results show that for a $C^{1}$ domain $S \subset R^{m}$ to be an invariant region we need at least the following.

Condition (c). $S$ is convex and for any $u \in \partial S$, the inward unit normal $\nu(u)$ at $u$ is a left-eigenvector of $D(x, t, u)$ and $M_{i}(x, t, u) \quad(i=1, \ldots, n)$, and $\nu(u) \cdot f(x, t, u) \geq 0$ for all $(x, t) \in \Omega \times(0, T)$.

Therefore in this paper, we shall always assume that Condition (c) holds.

\section{Preliminaries}

All materials discussed in this section can be found in the Appendix of Chapter 14 of [3], and they are included here for the reader's convenience.

First, let's recall some classical definitions. Suppose that $S$ is a $C^{2}$ domain in $\mathbf{R}^{m}$ with $\partial S \neq \phi$. For any $u \in \partial S$, let $\nu(u)$ denote the unit inner normal to $\partial S$ at $u$. For a fixed $u_{0} \in \partial S$, construct a coordinate system $\left(u_{1}, \ldots, u_{m}\right)$ such that the $u_{m}$-axis lies in the direction $\nu\left(u_{0}\right)$ and the origin is at $u_{0}$. Near $u_{0}, \partial S$ can be expressed by $u_{m}=\varphi\left(u_{1}, \ldots, u_{m-1}\right)$. Then the Gaussian curvature of $\partial S$ at $u_{0}$ is $\operatorname{det}\left[D^{2} \varphi(0)\right]$ and the principal curvatures of $\partial S$ at $u_{0}$ are the eigenvalues $k_{1}, \ldots, k_{m-1}$ of the matrix $\left[D^{2} \varphi(0)\right]$. Now if we rotate the coordinate frame with respect to the $u_{m}$ axis, we can let $u_{1}, \ldots, u_{m}$ axes lie on eigenvector directions corresponding to $k_{1}, \ldots, k_{m-1}$, respectively. We call such a new coordinate system a principal coordinate system at $u_{0}$. In this system $\left[D^{2} \varphi(0)\right]=\operatorname{diag}\left[k_{1}, \ldots, k_{m-1}\right]$.

For $u \in \mathbf{R}^{m}$, the distance function $d$ is defined by $d(u)=\operatorname{dist}(u, \partial S)$.

Lemma. Let $S$ be a $C^{k}$ domain in $\mathbf{R}^{m}, k \geq 2$ and $\partial S \neq \varnothing$. Then there exists an open (w.r.t the topology of $\bar{S}$ ) subset $G$ of $\bar{S}$ such that $G \supset \partial \Omega$, $d \in C^{2}(G)$, and for any $u \in G, \exists$ unique $y(u) \in \partial S$ such that $|u-y(u)|=$ $d(u)$ (i.e. $u=y(u)+\nu(y(u)) d(u)), \quad D d(u)=\nu(y(u)), \quad 1-k_{i}(y(u)) d(u)>0$ $(i=1, \ldots, m-1)$ where $k_{i}(y(u))(i=1, \ldots, m-1)$ are principal curvatures of $\partial S$ at $y(u)$. Moreover, for $u \in G$, at a principal coordinate system at $y(u)$,

$$
\left[D^{2} d(u)\right]=\operatorname{diag}\left[\frac{-k_{1}}{1-k_{1} d}, \ldots, \frac{-k_{m-1}}{1-k_{m-1} d}, 0\right] \text {. }
$$




\section{The MAIN RESUlT AND ITS PROOF}

In the rest of this paper, we assume that $u$ is a solution of $(*)$, and regard $D, a_{i j}$, and $M_{i}$ in $(*)$ as functions of $(x, t)$ only due to the compositions.

Theorem. Suppose that $D, a_{i j}$, and $M_{i}(1 \leq i, j \leq n)$ are locally bounded on $\Omega \times(0, T), D_{m \times m}$ and $\left(a_{i j}\right)_{n \times n}$ locally uniformly positive-definite on $\Omega \times$ $(0, T)$, and $f(x, t, u)$ is Lipschitz continuous in $u$ locally uniformly with respect to $(x, t)$ on $\Omega \times(0, T)$. Assume also that there exists a $C^{2}$ domain $S$ in $\mathbf{R}^{m}$ s.t. Condition (c) (in $\left.\S 1\right)$ is satisfied. Then if $u(\Omega \times(0, T)) \subset \bar{S}$ and there exists $\left(x^{*}, t^{*}\right) \in \Omega \times(0, T)$ s.t. $u^{*}=u\left(x^{*}, t^{*}\right) \in \partial S$, then $u\left(\Omega \times\left(0, t^{*}\right]\right) \subset \partial S$. Furthermore, if there exists $a x_{0} \in \partial \Omega$ and $0<t_{0}<T$ s.t. $\Omega$ satisfies the interior sphere condition at $x_{0}$ and $u$ is continuous at $\left(x_{0}, t_{0}\right)$ with $u\left(x_{0}, t_{0}\right) \in \partial S$, then either $u\left(\Omega \times\left(0, t_{0}\right]\right) \subset \partial S$ or $\nu\left(u\left(x_{0}, t_{0}\right)\right) \cdot \partial u / \partial \eta<0$. (if the directional derivative exists), where $\eta$ is any outward pointing direction to $\partial \Omega \times(0, T)$ at $\left(x_{0}, t_{0}\right)$.

Proof. Take a bounded open neighborhood $\Omega_{1} \subset \Omega$ of $x^{*}$ and $0<t_{1}<t^{*}$ s.t. $u\left(\Omega_{1} \times\left[t_{1}, t^{*}\right]\right) \subset G$ where $G$ is defined in the Lemma of $\S 2$.

Let $\mu(x, t, \nu)$ be the eigenvalue corresponding to eigenvector $\nu$ of $D(x, t)$ and $\lambda_{i}(x, t, \nu)$ be the eigenvalue of $M_{i}(x, t)$. Then on $\Omega_{1} \times\left[t_{1}, t^{*}\right]$

$$
\begin{aligned}
L= & \frac{\partial}{\partial t}-\mu(x, t, \nu(y(u(x, t)))) \sum_{i, j=1}^{n} a_{i j}(x, t) \frac{\partial^{2}}{\partial x_{i} \partial x_{j}} \\
& +\sum_{i=1} \lambda_{i}(x, t, \nu(y(u(x, t)))) \frac{\partial}{\partial x_{i}}
\end{aligned}
$$

is uniformly parabolic (for definitions of $\nu$ and $y(u)$, see $\S 2$ ).

Let $\bar{d}(x, t)=d(u(x, t))$. Then on $\Omega_{1} \times\left[t_{1}, t^{*}\right]$ we have

$$
\begin{aligned}
L \bar{d}= & D_{u} d(u) \frac{\partial u}{\partial t}-\mu(x, t, \nu(y(u))) \\
& \times \sum_{i, j=1}^{n} a_{i j}(x, t)\left(\sum_{\alpha, \beta=1}^{m} \frac{\partial^{2} d(u)}{\partial u_{\alpha} \partial u_{\beta}} \frac{\partial u_{\alpha}}{\partial x_{i}} \frac{\partial u_{\beta}}{\partial x_{j}}+\sum_{\alpha=1}^{m} \frac{\partial d(u)}{\partial u_{\alpha}} \cdot \frac{\partial^{2} u_{\alpha}}{\partial x_{i} \partial x_{j}}\right) \\
& +\sum_{i=1}^{n} \lambda_{i}(x, t, \nu(y(u))) \sum_{\alpha=1}^{m} \frac{\partial d(u)}{\partial u_{\alpha}} \cdot \frac{\partial u_{\alpha}}{\partial x_{i}} \\
= & D_{u} d(u) \frac{\partial u}{\partial t}-I(x, t)-\mu(x, t, \nu(y(u))) D_{u} d(u) \sum_{i, j=1}^{n} a_{i j}(x, t) \frac{\partial^{2} u}{\partial x_{i} \partial x_{j}} \\
& +\sum_{i=1}^{n} \lambda_{i}(x, t, \nu(y(u))) D_{u} d(u) \frac{\partial u}{\partial x_{i}}
\end{aligned}
$$

(continues) 


$$
\begin{aligned}
= & D_{u} d(u) \frac{\partial u}{\partial t}-D_{u} d(u) D(x, t) \sum_{i, j=1}^{n} a_{i j} \frac{\partial^{2} u}{\partial x_{i} \partial x_{j}}+D_{u} d(u) \sum_{i=1}^{n} M_{i} \frac{\partial u}{\partial x_{i}} \\
& -I(x, t) \\
= & D_{u} d(u) f(x, t, u)-I(x, t),
\end{aligned}
$$

where $I$ is defined by the second equality and in the third step we use the fact that $D_{u} d(u)=\nu(y(u))$ and Condition (c).

Now by Condition (c) again, $\nu(y(u)) f(x, t, y(u)) \geq 0$, i.e. $D_{u} d(y(u(x, t)))$. $f(x, t, y(u(x, t))) \geq 0$ on $\Omega_{1} \times\left[t_{1}, t^{*}\right]$. Hence we have

$$
\begin{aligned}
L \bar{d} \geq & D_{u} d(u(x, t)) f(x, t, u(x, t))-D_{u} d(y(u(x, t))) \\
& \cdot f(x, t, y(u(x, t)))-I(x, t) \\
= & \tilde{c}(x, t) \cdot(u(x, t)-y(u(x, t)))-I(x, t),
\end{aligned}
$$

where the $R^{m}$-vector function $\tilde{c}(x, t)$ is obtained by noticing $d \in C^{2}(G)$ and $f$ is Lipschitz in $u$. $\tilde{c}(x, t)$ is bounded on $\Omega_{1} \times\left[t_{1}, t^{*}\right]$. Since $u=y(u)+$ $\nu(y(u)) d(u)$, we have

$$
L \bar{d} \geq \tilde{c}(x, t) \nu(y(u(x, t))) d(u(x, t))-I(x, t),
$$

i.e.

$$
L \bar{d} \geq c(x, t) \bar{d}-I(x, t) \quad \text { on } \Omega_{1} \times\left[t_{1}, t^{*}\right],
$$

where $c$ is bounded.

Next, we prove $I \leq 0$ on $\Omega_{1} \times\left[t_{1}, t^{*}\right]$.

Fix $\left(x_{0}, t_{0}\right) \in \Omega_{1} \times\left[t_{1}, t^{*}\right]$. Since

$$
\sum_{\alpha, \beta=1}^{m} \frac{\partial^{2} d(u)}{\partial u_{\alpha} \partial u_{\beta}} \frac{\partial u_{\alpha}}{\partial x_{i}} \frac{\partial u_{\beta}}{\partial x_{j}}
$$

is invariant under any parallel translation and rotation of $u$ coordinate system, we assume that we work in a principle coordinate system at $y\left(u\left(x_{0}, t_{0}\right)\right) \in \partial S$. Then by the lemma

$$
D_{u}^{2} d\left(u\left(x_{0}, t_{0}\right)\right)=\operatorname{diag}\left[\frac{-k_{1}}{1-k_{1} d\left(u\left(x_{0}, t_{0}\right)\right)}, \ldots, \frac{-k_{1}}{1-k_{m-1} d\left(u\left(x_{0}, t_{0}\right)\right)}, 0\right]
$$

where $k_{1}, \ldots, k_{m-1}$ are the principal curvatures of $\partial S$ at $y\left(u\left(x_{0}, t_{0}\right)\right)$. Thus

$$
\frac{I}{\mu}\left(x_{0}, t_{0}\right)=\sum_{i, j=1}^{n} a_{i j}\left(x_{0}, t_{0}\right) \sum_{\alpha=1}^{m-1} \frac{-k_{\alpha}}{1-k_{\alpha} d\left(u\left(x_{0}, t_{0}\right)\right)} \frac{\partial u_{\alpha}}{\partial x_{i}}\left(x_{0}, t_{0}\right) \frac{\partial u_{\alpha}}{\partial x_{j}}\left(x_{0}, t_{0}\right),
$$

i.e.

(2)

$$
\frac{I}{\mu}\left(x_{0}, t_{0}\right)=\sum_{\alpha=1}^{m-1} \frac{-k_{\alpha}}{1-k_{\alpha} d\left(u\left(x_{0}, t_{0}\right)\right)} \sum_{i, j=1}^{n} a_{i j}\left(x_{0}, t_{0}\right) \frac{\partial u_{\alpha}}{\partial x_{i}}\left(x_{0}, t_{0}\right) \frac{\partial u_{\alpha}}{\partial x_{j}}\left(x_{0}, t_{0}\right) .
$$


Since $S$ is convex, $k_{\alpha} \geq 0,1 \leq \alpha \leq m-1$. Recall in the lemma that 1 $k_{\alpha}(u(u)) d(u)>0$ for $u \in G(\alpha=1, \ldots, m-1)$, so

$$
\frac{I}{\mu}\left(x_{0}, t_{0}\right) \leq 0 \text { on } \Omega_{1} \times\left[t_{1}, t^{*}\right] \text {. }
$$

In view of $(1)$, we have

$$
L \bar{d} \geq c(x, t) \bar{d} \quad \text { on } \Omega_{1} \times\left[t_{1}, t^{*}\right] .
$$

By the classical strong maximum principle, $\bar{d} \equiv 0$ on $\Omega_{1} \times\left[t_{1}, t^{*}\right]$, that is $u\left(\Omega_{1} \times\left[t_{1}, t^{*}\right]\right) \subset \partial S$. Thus we have proved that $u^{-1}(\partial S)$ is relatively open in $\Omega \times\left(0, t^{*}\right]$. Obviously $u^{-1}(\partial S)$ is relatively closed in $\Omega \times\left(0, t^{*}\right]$, hence $u\left(\Omega \times\left(0, t^{*}\right]\right) \subset \partial S$.

To prove the remaining part of the theorem, choose a bounded neighborhood $\Omega_{2}$ of $x_{0}$ which is relatively open in $\bar{\Omega}$ as well as a small $\delta>0$ such that $u\left(\Omega_{2} \times\left(t_{0}-\delta, t_{0}+\delta\right)\right) \subset G$. In the same way as above, we have for some bounded $C_{0}$

$$
L \bar{d} \geq C_{0}(x, t) \bar{d} \quad \text { on } \Omega_{2} \times\left(t_{0}-\delta, t_{0}+\delta\right) .
$$

Thus the classical boundary point lemma gives the desired result.

Remark 1. If the strict inequality in Condition (c) holds for all $(x, t) \in \Omega \times$ $(0, T)$, then there is no $\left(x^{*}, t^{*}\right) \in \Omega \times(0, T)$ s.t. $u\left(x^{*}, t^{*}\right) \in \partial S$.

The observations in [1] are still true for $(*)$, with slight modifications. Some of them are included in the following two remarks.

Remark 2. In the above theorem, $S$ can be the intersection of several $C^{2}$ domains $S_{j}$ which satisfy Condition (c). (In the case that $S_{j}$ 's meet at angles $<\pi / 2$, by this paper's proof, we just need $S$ to satisfy Condition (c).)

Remark 3. Combining (1) with $\bar{d} \equiv 0$, we have $I \geq 0$. So $I \equiv 0$. In view of (2) we have that if $k_{\alpha}>0$ for all $\alpha=1, \ldots, m-1, D_{x} u \equiv 0$. Thus we can add to the theorem that if $\partial S$ has positive Gaussian curvature everywhere, then $u$ is independent of $x$ when $0<t \leq t^{*}$.

Finally, concerning the elliptic systems corresponding to $(*)$, we have

Remark 4. The theorem holds for elliptic systems corresponding to $(*)$ with obvious modifications. Furthermore, it's also possible to extend the boundary point lemma for domains with corners (see $[5,6])$.

\section{ACKNOWLEDGMENTS}

I would like to thank Professors Wei-Ming Ni and Hans Weinberger for their interest in this work and constant encouragement. I also wish to thank Dr. Yi Li for his comments.

\section{REFERENCES}

1. H. Weinberger, Invariant sets for weakly coupled parabolic and elliptic systems, Rend. Mat. (7) 8 (1975), 295-310.

2. K. Chueh, C. Conley and J. Smoller, Positively invariant regions for systems of nonlinear diffusion equations, Indiana Univ. Math. J. 26 (1977), 373-392. 
3. D. Gilbarg and N. Trudinger, Elliptic partial differential equations of second order, 2nd ed., Springer-Verlag, Heidelberg, 1983.

4. W. Troy, Summary properties in systems of semilinear elliptic equations, J. Differential Equations 42 (1981), 400-413.

5. J. Serrin, A symmetry problem in potential theory, Arch. Rational Mech. Anal. 43 (1971), 304-318.

6. B. Gidas, W.-M. Ni and L. Nirenberg, Symmetry and related properties via the maximum principle, Comm. Math. Phys. 68 (1979), 209-243.

School of Mathematics, University of Minnesota, Minneapolis, Minnesota 55455 